\title{
Present and past mineral dust variations - a cross-disciplinary challenge for research
}

\author{
Ute Merkel', D.-D. Rousseau ${ }^{2,3}$, J.-B. W. Stuut ${ }^{1,4}$ and G. Winckler ${ }^{3,5}$
}

In its recently published report, the Intergovernmental Panel on Climate Change identified the role of mineral dust in the Earth system and the uncertainties it introduces to the total aerosol radiative forcing and climate projections as key topics for future research (WG 1, chapters 5, 7 and 9). Achieving a thorough understanding of feedbacks associated with eolian dust is a challenge for a number of Earth science disciplines as mineral dust processes operate on a wide range of spatial and temporal scales. On the other hand, studies of mineral dust contribute significantly to research on past climatic and environmental conditions enabled by dust preservation in different kinds of depositional paleoclimate archives.

Such work has been the focus of PAGES' recently concluded ADOM (Atmospheric Dust during the last glacial cycle: Observations and Modeling) working group, which was established in 2008 with the goal of combining reconstructions of climate and atmospheric circulation from terrestrial, marine and ice-core records with modern dust evidence and model simulations of past and present atmospheric circulation. To this end, ADOM considered processes ranging from the regional to (inter-) hemispheric scales and focused on fostering more detailed knowledge on dust-related dynamics. The idea of editing a dedicated PAGES Magazine on mineral dust was born during the $2^{\text {nd }}$ ADOM workshop held at MARUM in Bremen, Germany, in November 2011. This issue with contributions from workshop participants and colleagues from the ADOM community provides an overview of the science ADOM has focused on during recent years and highlights challenges to state-of-the-art dust research.

\section{Modern dust}

From a meteorological perspective the conditions governing the mobilization and entrainment of dust into the atmosphere (Fig. 1) and its long-range transport operate on daily to seasonal timescales. Schepanski et al. (p. 62) present an introduction to the modern mineral dust cycle and discuss on the basis of recent observational results how conditions for dust mobilization depend on daily meteorological conditions and on prevailing seasonal patterns. Lelli et al. (p. 64) show how seasonality impacts atmospheric aerosol content. Remote sensing of atmospheric aerosol content from satellites has now been carried out for more than three decades, which is sufficiently long to analyze interannual aerosol variations. However, developing algorithms to retrieve dust deposition information from the remotely sensed mineral dust content in the atmosphere is still a challenge (Lelli et al. p. 64). This is particularly relevant over the oceans where in-situ measurements of dust deposition are scarce (Fig. 2) but nevertheless required to put the dust deposition recorded in marine sediment into a quantitative perspective.

Seasonal dust fluctuations are also superimposed by longer-term variations on interannual-to-decadal timescales. Shao (p. 66) sheds light on the links between dust fluctuations and climate variability modes and trends, and presents recent regional dust modeling results for Asia and Australia. Altogether, these results emphasize the need for long-term monitoring of dust deposition. To that effect, the most striking long-term effort discussed in this issue is the dust recordings from J. Prospero's Barbados observatory. The observations now cover half a century and provide a deep insight into West African dust source variations and trans-Atlantic dust transport (Prospero $p$. 68). This record revealed for example a less pronounced correlation between precipitation in the Sahel region and dust deposited on Barbados than suggested previously.

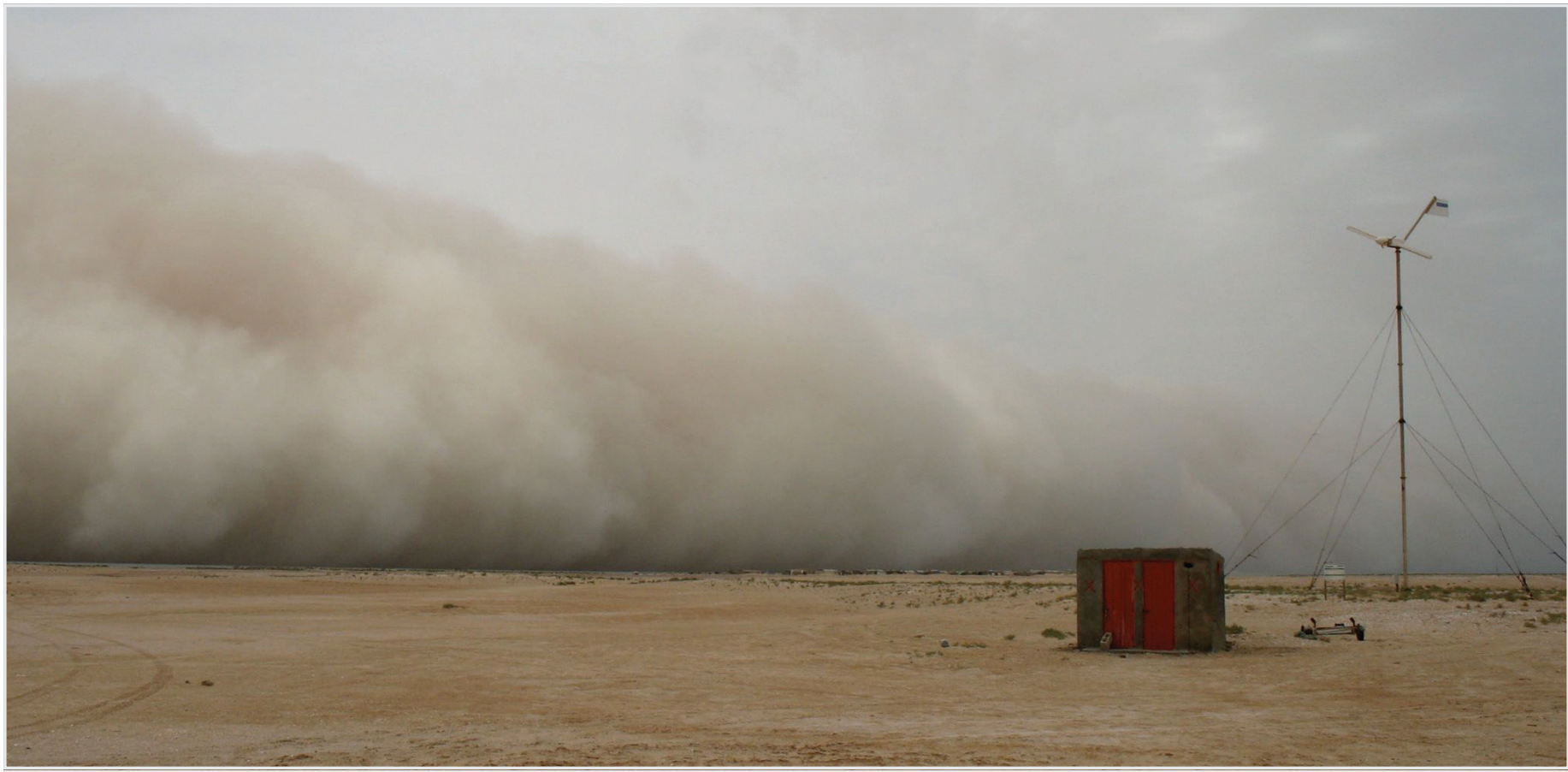

Figure 1: Dust storm in Iwik, Mauritania (photo by Jutta Leyrer). 


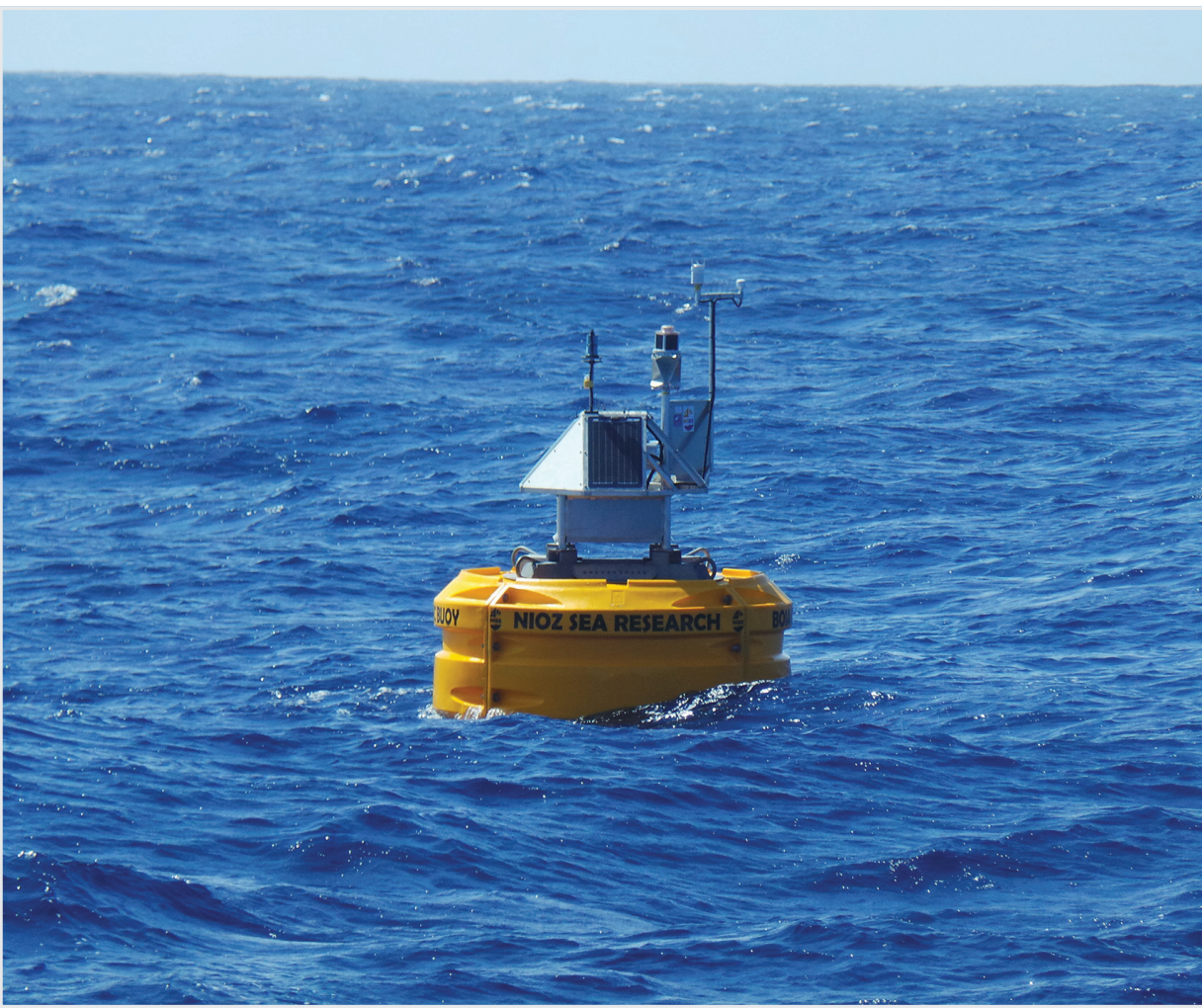

Figure 2: Dust collection at sea (photo by Jan-Berend Stuut).

In addition to analyses of the total amount of dust deposited such as those discussed above, understanding the evolution of particle-size distributions along the transport path is another important target of dust research. It requires that sectoral observational studies are coordinated towards a holistic source-to-sink approach, connecting research on near-source dynamics of surface dust emission, on size-selective transport processes in the atmosphere and on depositional processes including the sinking behavior of dust particles through the atmosphere and (ocean or lake) water column, and finally sediment formation. To achieve this, the different communities studying the dust cycle need to collaborate. Ideally, correlations between source area information and paleoclimatic records would emerge, taking into account the dust particle interactions and transformations on their way to the deposition site (Stuut et al. p. 70).

\section{Paleo archives of dust}

Looking into the past enables us to detect amplitudes, ranges, and timescales unseen in modern observational records. In spite of the many challenges associated with understanding atmospheric processes and dust particle dynamics on their source-to-sink pathway, paleo-dust reconstructions have provided elu cidating insights into the global dust cycle and its variations in the past. Paleoclimatic archives such as ice cores, terrestrial loess deposits, peat bogs, and marine sediments reveal direct information about variations of dust deposition processes over time. In addition they provide hints about dust-related facets of the Earth system, such as changes in vegetation cover, atmospheric circulation patterns and wind strength.

A central issue of paleo-dust studies is the question of provenance, i.e. what was the origin of the dust that eventually got deposited and preserved as sediment. The question becomes especially intriguing when paleo-dust archives are remote from established source areas such as it is the case for polar ice cores. These provide highly detailed information about gradual and abrupt climate change due to their high temporal resolution, but their interpretation requires assumptions about the long-range dust transport pathways. Based on the mineralogical and isotopic signatures of the dust, the Taklamakan desert of western China was recently identified as the main dust provenance region for Greenland ice cores (Bory p. 72). Mineral dust in East Antarctic ice cores has largely been attributed to Patagonian sources, in particular during glacial times. However, for the late Holocene, Australia and also Antarctica itself have now been identified as secondary dust sources to Antarctica (Vallelonga p. 74). Furthermore, dust signatures at West and East Antarctic deposition sites have been shown to be impacted by topographic elevation effects (Koffman and Kreutz p. 76). Similarities detected recently in the geochemical fingerprint of Australian and Southern South American dust (Gili and Gaiero p. 78) further complicate the interpretation of dust in Antarctica.

A large amount of mineral dust is deposited over the open ocean and leaves an imprint in marine sediments. Recent methodological developments have added non-destructive fine-scale elemental scanning and geochemical fingerprinting methods to the portfolio of sediment core analysis. The gain in information detail reveals new insight into dust provenance and transport mechanisms, as demonstrated with a marine sediment core off southern Australia (De Deckker p. 80). Reconstructing dust deposition over the ocean is of particular interest because of the potential role of dust input in fertilizing high-nutrient, low-chlorophyll regions of the surface ocean with the micronu trient iron, thereby stimulating phytoplankton growth and organic carbon export to the deep ocean. Iron fertilization as traced in marine sediments from the Southern Ocean (Martínez García and Winckler p. 82) may help explain variations in atmospheric $\mathrm{CO}_{2}$ over past glacial climate cycles, and help to asses the potential of artificial iron fertilization as geo-engineering strategy.

Understanding the variability of the dust cycle in the past is closely tied to knowledge about past atmospheric circulation. The continental loess deposits at mid-latitudes around the world provide information about paleowind direction and intensity. Recent examples demonstrate that loess properties such as coarse-to-fine particle ratios and alterations in paleosol-loess sequences can provide insight into glacial-interglacial variations in eolian particle transport (Muhs et al. p. 84). Furthermore, an overview of global loess deposits and a comparison between climate model results and loess records in Europe highlights the potential for studying the role of changes in the seasonal cycle for the millennial-scale abrupt changes of Marine Isotope Stages 2 and 3 (Rousseau and Sima p. 86). During the past decade, peat bogs and the mineral dust they contain have turned out to be a new valuable natural archive for reconstructing the role of dust in climate change and during abrupt events, in particular during the Holocene (De Vleeschouwer et al. p. 88).

\section{Encouraging collaboration}

As emphasized by the contributions in this dust issue of PAGES Magazine, the evidence from proxies and model simulations about dust variations in the past is increasing rapidly. These are good conditions to foster cross-disciplinary approaches that combine more advanced characterizations of dust sources with the dust signatures at deposition sites. Concerted efforts are needed to produce quantitative proxy records and a synopsis of available records. Such an improved data basis would not only benefit the paleo-dust reconstruction community but climate modelers, who require quality-controlled globally gridded datasets as model input or for meaningful model-data comparisons. As an example of such concerted efforts, this issue provides news from the DIRTMAP initiative (Maher and Leedal, p. 90) and the new PAGES working group, DICE (Dust Impacts on Climate and Environment; Winckler and Mahowald, p. 61). DICE will build on the successful legacy of ADOM and provide a collaborative platform to build a tight and well-coordinated link from (paleo) observations to (paleo) climate modeling.

\section{AFFILIATIONS}

'MARUM - Center for Marine Environmental Sciences and Faculty of Geosciences, University of Bremen, Germany 'Laboratoire de Météorologie Dynamique \& CERES-ERTI, CNRS - Ecole Normale Supérieure, Paris, France ${ }^{3}$ Lamont-Doherty Earth Observatory, Columbia University, Palisades, USA

${ }^{4} \mathrm{NIOZ}$ Royal Netherlands Institute for Sea Research, Texel, The Netherlands

${ }^{5}$ Department of Earth and Environmental Sciences, Columbia University, New York, USA

\section{CONTACT}

Ute Merkel: umerkel@marum.de 